tions in $\mathrm{mg} / \mathrm{dl}$ instead of $\mathrm{mmol} / \mathrm{l}$ and errors such as in table 7 , p 461 which reports a gene frequency of familial hypercholesterolaemia of 1:1000 instead of $1: 500$

The chapter on the genetics of the human apolipoproteins by Jan Breslow is well written, by an established expert in the field. However, due to the limitations outlined previously, there is no mention of the recent reports suggesting that specific apolipoprotein restriction fragment length polymorphisms may be genetic markers for susceptibility to develop obstructive coronary artery disease. Furthermore, there is no discussion of current clinical controversies regarding the value of measuring plasma apoproteins such as apo A-I, A-II, and B in predicting the presence of significant coronary artery disease.

In summary, this volume provides a good reference to the current concepts in lipid metabolism, but will appeal mainly to the non-clinician and basic scientists interested in this field.

Alan Rees

\section{Chorionic Villi Sampling}

Contributions to Obstetrics and Gynaecology No 15. Series editor P J Keller. Edited by G Pescia and H Nguyen The. (Pp 116; £31.40.) Basle: Karger. 1986.

There are now several useful books about chorionic villus sampling so one may ask, do we really need another just yet? The answer seems to be yes. This is a worth while little volume containing short, clear, and well illustrated articles by members of several European groups with considerable experience and a pleasing scientific approach. It contains something of value for most workers in the field and will be read with pleasure much more widely. It will prove particularly suitable for the audience that it is aimed at, namely obstetricians.

It starts with a review of obstetric approaches for CVS by Bruno Brambati, with particular emphasis on obstetric complications, and a reassuring short term follow up of the newborn babies in the larged Milan series. There is also a careful description of: the problems and solutions of karyotyping chorionicos villus material by $G$ Terzoli and $G$ Simoni, with upo to date recommendations on how to maximise theo number of mitotic figures available in short term (48क hour) chorionic villus cultures.

B Gustavii has three short articles in which he explains that he abandoned CVS under direct vision ${ }^{\text {s }}$ because it carries a greater risk of spontaneouss abortion than other approaches; describes his simple $\vec{\psi}$ method of transabdominal chorionic villus sampling ${ }_{\mathscr{\sigma}}$ and discusses reasons for the high frequency of non $\overline{3}$ viable pregnancies found by ultrasound in womerp presenting with the request for CVS.

An obstetrician, a clinical geneticist, and $\dot{\vec{a}}$ cytogeneticist describe their experience in Lausanne in three chapters that make easy reading because of their anecdotal nature. Three articles from Paris, on CVS using biopsy forceps, on a method for selective early abortion in multiple pregnancies, and on the $z$ application of molecular methods for prenatal diag울 nosis of the haemoglobinopathies are particularlys welcome, as we might easily miss such articles in the्ष French literature.

In addition, there is an interesting article bye Kleijer from Rotterdam on the present state of payen

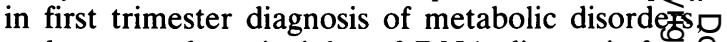
and one on the principles of DNA diagnosis frem D N Cooper of the UK.

The book provides a good technical introduction to CVS. Its shortcoming is the absence of ax discussion of the principles of evaluating the shor and long term risks of the new procedure. Mosê巳 practitioners are likely to become involved in a national or international collaborative register, or better, in a randomised controlled trial. For those entering the field, this is one of the most difficults scientific aspects to come to terms with. A shor crisp article explaining the need for, and the practitioner's moral responsibility to participate in good scientific follow up studies would have been particularly valuable. 